\title{
Structure, properties, and MEMS and microelectronic applications of vanadium oxides
}

\author{
ROBERT B DARLING* and SHIHO IWANAGA \\ University of Washington, Department of Electrical Engineering, Box 352500, \\ Seattle, WA 98195-2500 USA \\ e-mail: bruced@u.washington.edu
}

\begin{abstract}
Vanadium oxides have for many decades attracted much attention for their rich and unique physical properties which pose intriguing questions as to their fundamental origins as well as offering numerous potential applications for microelectronics, sensors, and microelectromechanical systems (MEMS). This paper reviews the unique structure and properties of the two most common vanadium oxides which have entered into microfabricated devices, $\mathrm{VO}_{2}$ and $\mathrm{V}_{2} \mathrm{O}_{5}$, and some of the past and future device applications which can be realized using these materials. Two emerging new materials, sodium vanadium bronzes and vanadium oxide nanotubes are also discussed for their potential use in new microelectronic devices.
\end{abstract}

Keywords. Vanadium oxides; microbolometers; thermoelectrics; sensors; MEMS.

\section{Introduction}

The transition metal oxides offer a wealth of unique physical, chemical, electronic, optical, magnetic, and thermal properties which have occupied scientists and engineers for decades. The vanadium oxides offer many convenient physical effects which can be harnessed for different microelectronic device applications. Vanadium, with its $[\mathrm{Ar}] 3 \mathrm{~d}^{3} 4 \mathrm{~s}^{2}$ electronic configuration, provides unique $3 d$-orbital mixing in the chemical bonds that it makes, and this leads directly to its multivalent character $(+2,+3,+4,+5)$ which can be affected by crystal structure, habit, defects, and external redox electron transfer. Because vanadium occurs naturally in one predominant isotope $\left(\mathrm{V}^{51}-99.76 \%, \mathrm{~V}^{50}-0.24 \%\right)$, its compounds have very little isotopic disorder, which leads to stable and repeatable properties at both the nano- and mesoscale. Vanadium forms a very large number of oxides, although only a select few provide the stability needed for microelectronic devices, and these include $\mathrm{VO}, \mathrm{V}_{2} \mathrm{O}_{3}, \mathrm{VO}_{2}, \mathrm{~V}_{2} \mathrm{O}_{5}$, and $\mathrm{VO}_{3} . \mathrm{VO}_{2}$ has been intensively studied for its metal-insulator phase transition (MIT) that occurs at a very technologically useful temperature of $67^{\circ} \mathrm{C}$. A similar MIT has been observed in $\mathrm{V}_{2} \mathrm{O}_{3} . \mathrm{V}_{2} \mathrm{O}_{5}$ has also been intensely studied for its electrochromic and thermochromic effects which can be harnessed for electro-optical device applications. The unique structure

*For correspondence 


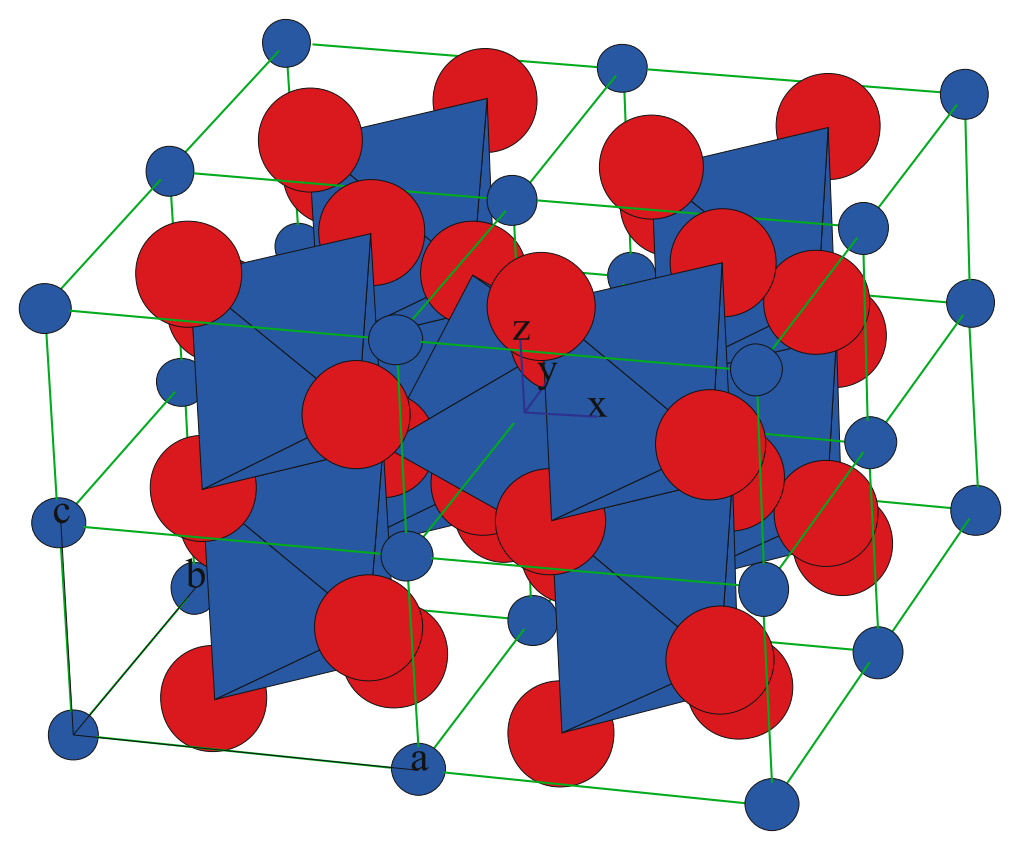

Figure 1. Crystal structure of $\mathrm{VO}_{2}$. Smaller blue circles are vanadium atoms; larger red circles are oxygen atoms. $\mathrm{VO}_{6}$ octahedra are shown in blue.

and properties of both of these oxides will be briefly reviewed, along with their more promising device applications. New materials are also being developed in the vanadium oxide family. Several of these will also be discussed in terms of their future promise for applications to microelectronic devices, sensors, and systems.

\section{Vanadium dioxide, $\mathrm{VO}_{2}$}

\subsection{Crystal structure}

In its higher temperature metallic phase (above $67^{\circ} \mathrm{C}$ ), $\mathrm{VO}_{2}$ has a tetragonal crystal structure, similar to that of the mineral rutile, with each vanadium coordinated to six nearest oxygens, forming octahedra. These octahedra are symmetrical and form edge-sharing chains along the $c$-axis of the crystal, with the vanadium atoms in a nominal +4 oxidation state. This is usually referred to as the metallic R-phase, and it has lattice parameters of $a=b=4.553^{\prime}$ and $c=2 \cdot 849^{\prime}$ (Kucharczyk \& Niklewski 1979). One of the principal features of this lattice is that the $c / a$ ratio is rather small in comparison to other rutile-structured compounds, which places adjacent vanadium atoms closer together along the $c$-axis. This crystal structure is illustrated in figure 1.

\subsection{Metal-insulator phase transition (MIT)}

When cooled below $67^{\circ} \mathrm{C}, \mathrm{VO}_{2}$ undergoes a sharp and reversible phase transition into a monoclinic crystal structure with $a=5 \cdot 384^{\prime}, b=4.526^{\prime}, c=5.574^{\prime}$, and $\gamma=122.626^{\circ}$ (Kucharczyk \& Niklewski 1979). The vanadium atoms dimerize, which leads to an alternating 


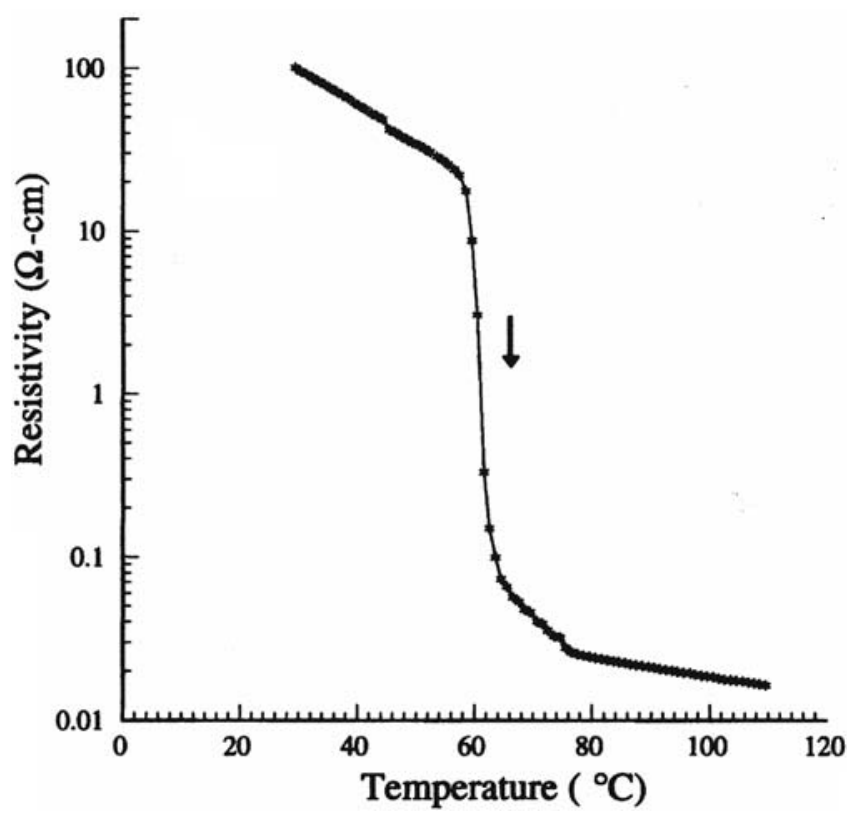

Figure 2. Electrical resistivity near the metal-insulator transition (MIT) for $\mathrm{VO}_{2}$. From Jerominek (1993).

zig-zag pattern along the $c$-axis. This breaks the existing symmetry of the metallic phase, and causes a doubling of the unit cell, also along the $c$-axis (Kawada et al 1971). The previously symmetrical $\mathrm{VO}_{6}$ octahedrons become distorted, and the associated mixing of the $3 d$-orbitals results in a shift in the energy band structure of the material. The overlapping bands of the metallic phase become split apart to produce an energy bandgap, and the material thus transitions to an insulating phase, usually referred to as the $M_{1}$ phase.

While the existence of this MIT has been known for decades, there are still fundamental questions about the driving forces behind it, specifically, whether the change in crystal structure prompts the change in electronic structure, or vice-versa (Adler et al 1967; Biermann et al 2005; Haverkort et al 2005; Narayan \& Bhosle 2006). Recent reports have shown that the phase transition requires at least $80 \mathrm{fs}$, which is about half of a period of a lattice vibration, and indicative of a transition that is driven by electron-electron correlations, confirming the original suspicions that $\mathrm{VO}_{2}$ followed the Mott-Hubbard model (Cavalleri et al 2004a, $2004 \mathrm{~b}, 2005)$. However, there still remain questions as to how the deformations of the $\mathrm{VO}_{6}$ octahedra fit into this picture, as well as more specific details concerning the evolution of the changes in the energy bands.

Across the MIT, the electrical resistivity of $\mathrm{VO}_{2}$ changes by over a factor of $10^{2}$ in the span of less than one degree, falling from about $20 \Omega$-cm in the insulating phase to about $0.08 \Omega$-cm in the metallic phase, as shown in figure 2 (Jerominek 1993). This sharp change in resistivity makes the MIT of $\mathrm{VO}_{2}$ an exquisite temperature sensor in the vicinity of the MIT. It has been found that the MIT temperature can be increased by chromium doping $\left(\sim 3^{\circ} \mathrm{C} / \mathrm{at} . \% \mathrm{Cr}\right)$ in which $\mathrm{Cr}$ substituting for $\mathrm{V}$ reduces the size of the unit cell (Pan et al 2004). $\mathrm{VO}_{2}$ also exhibits thermochromism and electrochromism, both of which can be modified by the deposition conditions (Jin et al 1997) and by lithium doping (Kahn et al 1991). These effects have been exploited to achieve high-contrast optical storage (Balberg \& Trokman 1975). 


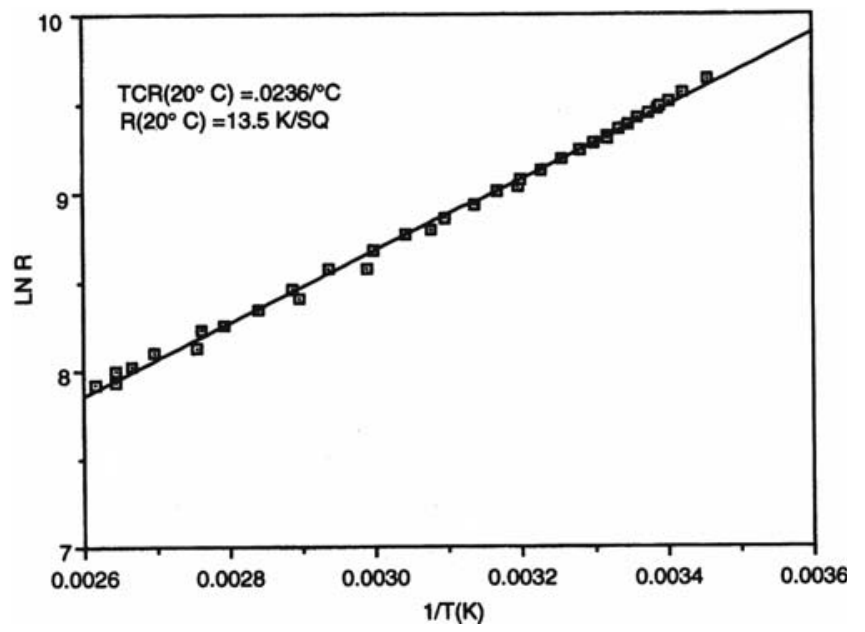

Figure 3. Electrical resistivity versus temperature for mixed $\mathrm{VO}_{x}$ oxides. From Wood \& Stelzer (1992).

\subsection{Application to infrared microbolometer arrays}

One of the more technologically significant uses for vanadium oxides are in the fabrication of microbolometer arrays for infrared imaging. Imaging and detection of mid-infrared radiation, nominally around $\lambda=10 \mu \mathrm{m}$, has traditionally been achieved through direct photon excitation of electron-hole pairs in narrow bandgap semiconductors such as $\mathrm{InSb}$ and $\mathrm{Hg}_{x} \mathrm{Cd}_{1-x} \mathrm{Te}$; however, the otherwise high intrinsic carrier concentration for these materials necessitates the use of cryogenic cooling to at least $77 \mathrm{~K}$ or below. Room temperature uncooled IR imaging remained elusive until the concept of micromachined thermally isolated structures were proposed as bolometer supports in the late 1970s.

The development of micromachined support structures for infrared microbolometer arrays was started by Wood's group at Honeywell in 1982 (Wood et al 1992). Silicon nitride membranes were initially used as supports over top of sacrificially etched cavities to provide a thermally isolated platform for the bolometer material. The initial materials that were used for bolometers were $\mathrm{Pt}$ and $\mathrm{Ni}: \mathrm{Fe}$ with temperature coefficients of resistance (TCRs) of $+0 \cdot 0018 / \mathrm{K}$ and $+0 \cdot 0023 / \mathrm{K}$, respectively. While these materials have good TCR values for bolometer use, they suffer from having rather low resistivity, which requires many squares of a serpentine pattern to be created to achieve a nominal bolometer resistance of around $10-50 \mathrm{k} \Omega$, and they have comparatively high $1 / f$ noise spectra, which limits the ultimate noise-effective temperature difference (NETD) which can be achieved. Vanadium oxides were chosen as a better bolometer material because of their combination of high TCR, high resistivity, good IR absorption characteristics, and low $1 / f$ noise. In addition, the vanadium oxides offer good sputtering characteristics, and good passivation characteristics in conjunction with silicon nitride.

The $67^{\circ} \mathrm{C}$ MIT of $\mathrm{VO}_{2}$ provides an extremely sharp change in resistivity versus temperature, but this transition is actually too sharp to use effectively in a microbolometer array, as this would require extremely demanding thermal control to bias the material in the middle of this transition region. Instead, the Honeywell group took the approach of using a mixed oxide system of $\mathrm{V}_{2} \mathrm{O}_{3}, \mathrm{VO}_{2}$, and $\mathrm{V}_{2} \mathrm{O}_{5}$ to create a stable microbolometer material with a high TCR and high resistivity, as shown in figure 3 (Wood \& Stelzer 1992).

Over 1982-1992, there were no reports of room temperature uncooled IR imager development, due to the on-going work at Honeywell being a classified US project. In 1992, this 


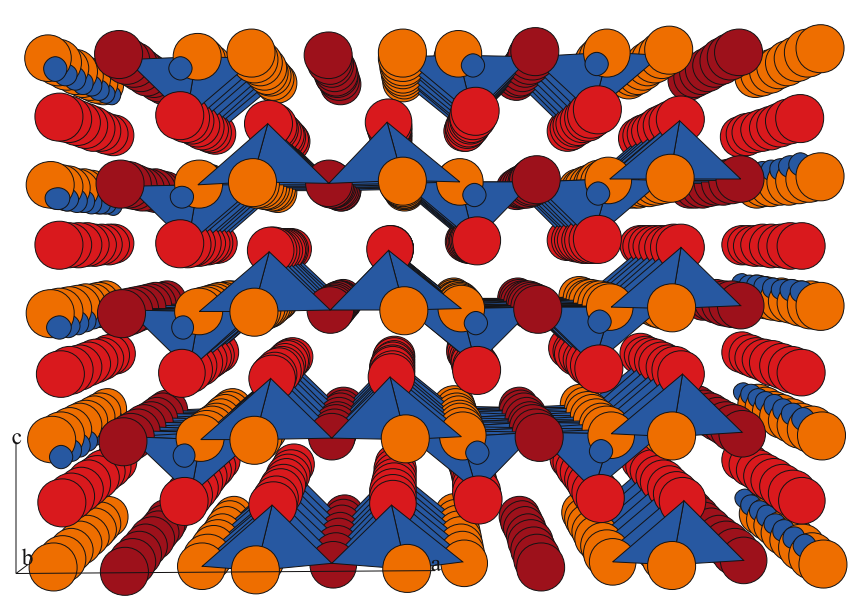

Figure 4. Crystal structure of $\mathrm{V}_{2} \mathrm{O}_{5}$. Smaller blue circles are vanadium atoms; larger red circles are vanadyl oxygens; larger orange circles are chain oxygens; larger brown circles are bridge oxygens. Each van der Waals layer in the $a-b$ plane is comprised of alternating double chains of $\mathrm{VO}_{5}$ pyramids, shown in blue.

work was declassified, and was first presented by Wood and coauthors Wood et al (1992). They disclosed a $230 \times 336$ pixel imager with a 70 percent fill factor, $30 \mathrm{~Hz}$ frame rate, and $8-14 \mu \mathrm{m}$ spectral window. The pixels were $50 \mu \mathrm{m}$ square, with a resistance of $20 \mathrm{k} \Omega$, a TCR of $-0.0023 / \mathrm{K}$, and a noise-effective temperature difference (NETD) of $0.039^{\circ} \mathrm{C}$. The pixels were created using a two-layer design in which the row and column interconnects were created at a lower level on the substrate and the micromachined silicon nitride supports with a $\mathrm{VO}_{x}$ bolometer were created over top (upper level). This basic design has persisted for over 15 years now, and has been commercialized in a number of formats. Amber Engineering, Inc., introduced a hand-held, portable IR imaging camera using this technology in 1995, and more recently in 2005, John Fluke Mfg. Co. has introduced a number of models of handheld, portable IR imaging cameras which have become the industry standards. Today, these imagers are used routinely for HVAC and building heating/cooling analysis, in machinery maintenance, and in microelectronic thermal diagnostics.

Critical to the success of the room-temperature, uncooled microbolometer at Honeywell was the development of a stable, high TCR bolometer material using the mixed vanadium oxides. This example illustrates the successful research and development of the vanadium oxides toward a commercial product which has significantly advanced the field of infrared detection and imaging.

\section{Vanadium pentoxide, $\mathbf{V}_{2} \mathbf{O}_{5}$}

\subsection{Crystal structure}

Unlike $\mathrm{VO}_{2}$ which exhibits multiple crystal phases, $\mathrm{V}_{2} \mathrm{O}_{5}$ exists in only one known crystal structure. It is an orthorhombic crystal with $a=11.51^{\prime}, b=4 \cdot 37^{\prime}$, and $c=3.56^{\prime}$ and a $P_{m m n}$ space group (Eyert $\&$ Höch 1998). It is fundamentally comprised of $\mathrm{VO}_{5}$ pyramids which form alternating double chains along the $b$-axis. Alternating double chains of pyramidsup/pyramids-down are connected laterally by bridging oxygens to form a sheet or ribbon in the $a-b$ plane. The planes themselves are connected by van der Waals bonds, and this looser bonding creates easy cleavage along these planes. The crystal structure is illustrated in figure 4, and gives rise to three non-equivalent oxygen positions. The $\mathrm{O}_{v}$ or vanadyl oxygens are singly coordinated to the $\mathrm{V}$ atoms and lie closest to and directly above or below the 
$\mathrm{V}$ atoms in the $c$-directions. The $\mathrm{O}_{c}$ or chain oxygens are triply coordinated and create the primary chain linkages along the $b$-axis. The $\mathrm{O}_{b}$ or bridge oxygens are doubly coordinated and connect the chains laterally in the $a$-directions. The crystal structure of $\mathrm{V}_{2} \mathrm{O}_{5}$ creates very robust ribbons along the $b$-axis direction, but otherwise, the lattice is fairly open and permeable to smaller intercalating cations. One of the primary uses of $\mathrm{V}_{2} \mathrm{O}_{5}$ is as a highcapacity ion storage medium in Li-ion batteries. The surfaces of the van der Waals planes are polar and capable of being hydrated (Hermann et al 1999). As a powder, $\mathrm{V}_{2} \mathrm{O}_{5}$ does not readily dissolve in water, but at elevated temperatures, the ribbon surfaces can be hydrated, after which the ribbon becomes readily soluble in water and takes the form of a gel.

Vanadium pentoxide is a semiconductor with a bandgap of $2 \cdot 3-2.4 \mathrm{eV}$. The bandgap is an indirect transition from the split-off oxygen $2 p$ band up to the vanadium $3 d$ band. Electronic conduction in $\mathrm{V}_{2} \mathrm{O}_{5}$ is highly anisotropic, with conduction within the $a-b$ planes considerably higher than conduction perpendicular to these planes. The crystal structure of $\mathrm{V}_{2} \mathrm{O}_{5}$ places the vanadium atoms into a nominal +5 valence state, but thermal agitation and crystal defects work to create a small concentration of +4 valent vanadium atoms. Electronic conduction occurs by means of electron hopping which is equivalent to the motion of these +4 valent vanadium states. The hopping is much easier within the $a-b$ planes, due to the shorter distances between vanadium atoms, than between the planes, leading to the in-plane/out-of-plane conduction anisotropy. The +4 valent vanadium state also creates a local lattice distortion which must travel along with the hopping electron. The combination of the electron and its accompanying lattice distortion is a polaron, and electronic conduction in $\mathrm{V}_{2} \mathrm{O}_{5}$ is dominated by this small polaron transport. In comparison to more conventional semiconductor conduction, the charge carriers in $\mathrm{V}_{2} \mathrm{O}_{5}$ are negative charges with a greatly reduced effective mobility, and which carry with them the energy of the electron and the energy of the lattice distortion.

\subsection{Sol-gel fabrication processes}

The high aqueous solubility of hydrated $\mathrm{V}_{2} \mathrm{O}_{5}$ allows its use in solution-based microfabrication methods, which are fundamentally inexpensive, both in terms of materials and equipment, scalable to large areas, and do not involve any toxic or otherwise dangerous chemicals. Solgel techniques have proven to be the most versatile, and of these the melt-quench technique has yielded good and reproducible results on a variety of substrates. A typical melt-quench process would start by melting a $\mathrm{V}_{2} \mathrm{O}_{5}$ precursor powder in a platinum or ceramic crucible and allowing it to stabilize at $900-1000^{\circ} \mathrm{C}$ for 30 minutes (Bullot et al 1980). $\mathrm{V}_{2} \mathrm{O}_{5}$ melts at $670-685^{\circ} \mathrm{C}$. The melt is then slowly poured into stirred deionized water over a period of 30 minutes, which creates long fibres or ribbons of $\mathrm{V}_{2} \mathrm{O}_{5}$ ( $b$-axis chains) with hydrated surfaces. The result is an orange-coloured gel which can then be applied to substrates by any of a number of methods, including dip coating, spin coating, casting, spraying, or capillarydriven wetting. After application, the film is slowly dehydrated and then annealed at $250^{\circ} \mathrm{C}$ for 10 minutes. This process usually causes the van der Waals planes to lay flat against the surface. X-ray diffraction analysis of the films reveals that, indeed, the $c$-axis of the ribbons becomes aligned normal to the substrate surface, although the $a$ - and $b$-axes can be randomized. Depending on the concentration of the gel, the resulting thickness of the $\mathrm{V}_{2} \mathrm{O}_{5}$ film can be rather thin, typically 50-250 nm. To achieve greater thicknesses, multiple coating and annealing steps can be performed. A final firing at $400-500^{\circ} \mathrm{C}$ completes the process.

There are other methods for creating $\mathrm{V}_{2} \mathrm{O}_{5}$ thin flims, such as RF sputtering, but these typically do not produce good crystallinity or $c$-axis oriented films, since the sputtering process deposits small clusters, not long ribbons. Sol-gel techniques have thus far offered the most versatile and repeatable method of $\mathrm{V}_{2} \mathrm{O}_{5}$ thin film fabrication. Further, the sol-gel techniques 


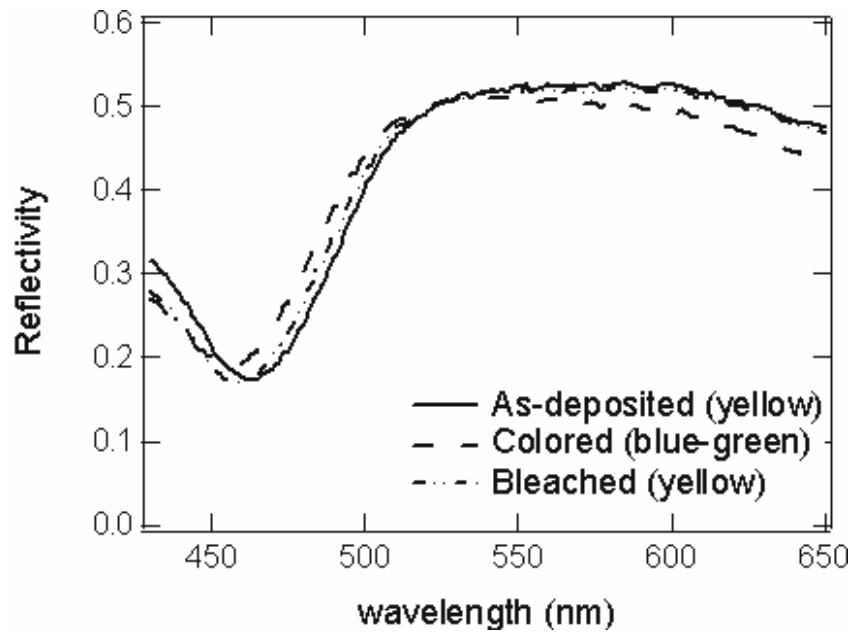

Figure 5. Optical reflectivity of $\mathrm{V}_{2} \mathrm{O}_{5}$ thin films modified by AFM nanolithography. From Iwanaga et al (2005).

have been particularly well-suited for creating a wide variety of unique nanostructure shapes, when adapted to hydrothermal synthesis methods with additional molecular modifiers or intercalating molecules.

\subsection{Electrochromism}

The property most associated with $\mathrm{V}_{2} \mathrm{O}_{5}$ is its electrochromic effect which can produce vibrant colours in the material. Electrochromic effects are persistent and reversible changes in optical properties that result from the injection or extraction of charge, and are exhibited by a large number of the transition metal oxides. Cathodic electrochromism refers to a decrease in transmittance caused by charge injection, e.g. in Ti and $W$ oxides, while anodic electrochromism refers to an increase in transmittance caused by charge injection, e.g. in $\mathrm{Cr}$, $\mathrm{Mn}$, and $\mathrm{Fe}$ oxides. Vanadium pentoxide is somewhat unique by exhibiting a transmittance increase in the near-UV and blue part of the visible spectrum and a simultaneous transmittance decrease in the near-IR and red part of the visible spectrum (Cogan et al 1989; Talledo et al 1991). Charge injection in $\mathrm{V}_{2} \mathrm{O}_{5}$ can be accomplished using electrochemical charge transfer reactions with $\mathrm{H}^{+}, \mathrm{Li}^{+}$, or $\mathrm{Na}^{+}$which intercalate these ions into the van der Waals planes of the crystal. The resulting intercalations are known to monotonically increase the $c$ - and decrease the $a$-lattice parameters, although somewhat nonlinearly with concentration. For crystalline $\mathrm{V}_{2} \mathrm{O}_{5}$, intercalation with $\mathrm{Li}^{+}$to levels of around $15 \mathrm{mC} / \mathrm{cm}^{2}$ causes an increase in the energy bandgap from about $2.38 \mathrm{eV}$ to about $2.75 \mathrm{eV}$, corresponding to an equivalent formula of $\mathrm{Li}_{2 \cdot 2} \mathrm{~V}_{2} \mathrm{O}_{5}$. Increased absorption around $1.0 \mu \mathrm{m}$ is thought to arise from vanadyl oxygen vacancies $\left(\mathrm{V}_{\mathrm{OV}}\right)$ which create $\mathrm{V}^{4+}$ pairs and localized $3 d^{1}$ electron states within the bandgap (Talledo \& Granqvist 1995). The $\mathrm{V}^{4+}$ states create small polarons which can account for the increased near-IR absorption.

Electrochromism has been investigated as a mechanism for creating smart windows with adjustable reflectivity for use in buildings, automobiles, aircraft, and space satellites. More recently, it has been found that electrochromic effects in $\mathrm{V}_{2} \mathrm{O}_{5}$ can be created by charge injection from an atomic force microscope (AFM) tip (Iwanaga et al 2005). This offers a new type of AFM nanolithography in which the patterns can be both locally written and erased with nanometer scale resolution, and which are persistent for up to many months in air. Existing AFM nanolithography has used the tip to locally oxidize clean, bare surfaces 
of $\mathrm{Si}, \mathrm{Al}$, and $\mathrm{Ti}$; however, these patterns are short lived, as the bare surrounding areas also oxidize on exposure to air, often within only a few minutes. By using a thin film such as $\mathrm{V}_{2} \mathrm{O}_{5}$ which is already in nearly its highest possible oxidation state, the problem of pattern fading upon exposure to air can be eliminated. Figure 5 shows the reflectivity spectrum of $\mathrm{V}_{2} \mathrm{O}_{5}$ which has been initially deposited on to a $\mathrm{Cr}$ layer on a Si substrate. The film starts out with a yellow colour, and after writing with the AFM with a tip bias of $+7.0 \mathrm{~V}$, relative to the substrate, the film is transformed into a blue-green colour. By re-writing the colourized zone with a tip bias of $-6.0 \mathrm{~V}$, the colour can be reversed back to yellow. The AFM writing was performed under room environment conditions, and the normal humidity of the air creates a water condensation droplet at the AFM tip. This water droplet forms a source of $\mathrm{H}^{+}$, which are believed to be the intercalating species. Tip currents of about $10 \mathrm{nA}$ are used for initial writing of the patterns, but after the voltage reaches $+3.0 \mathrm{~V}$, the tip current abruptly falls to the $10 \mathrm{pA}$ level. The AFM nanolithography process thus produces high-contrast changes in the optical and electrical properties of the $\mathrm{V}_{2} \mathrm{O}_{5}$ film, both of which could be potentially developed into a high-density digital storage technology.

\subsection{Thermoelectric properties}

Transition metal oxides are not normally associated with good thermoelectric properties. Traditional thermoelectric materials are ones with high atomic weight and narrow bandgap, such as $\mathrm{Bi}_{2} \mathrm{Te}_{3}, \mathrm{Sb}_{2} \mathrm{Te}_{3}, \mathrm{PbTe}$, and $\mathrm{SiGe}$. For energy conversion processes, such as DC electric current generation or solid-state refrigeration, the figure-of-merit for a thermoelectric material is its ZT value, where $Z=\sigma S^{2} / \kappa, \sigma$ is the electrical conductivity, $S$ is the Seebeck coefficient, and $\kappa$ is the thermal conductivity. $T$ is the average temperature of the hot and cold sides. Most conventional thermoelectric materials have ZT values of about $0 \cdot 5$. A ZT value of about 3.0 or higher is technologically very significant, because at this level the energy efficiency of thermoelectric refrigeration becomes equivalent to conventional refrigeration using rotating machinery and refrigerant gases. If a low-cost, easily synthesized material with a ZT value of greater than 3 could be found, its impact would be to displace conventional refrigeration equipment with no-moving-parts, highly reliable, smaller, and lighter weight thermoelectric refrigeration. Recently, a few materials with ZT figures approaching 3 have been discovered, but these have so far been semiconductor superlattices which are far from economical to manufacture or possible to create in large sizes.

Recently, it has been found that $\mathrm{V}_{2} \mathrm{O}_{5}$ thin films exhibit an anomalously high ( $n$-type) Seebeck coefficient of about $-540 \mu \mathrm{V} / \mathrm{K}$ (Iwanaga et al 2007). This can be attributed to the small polaron transport process, in which the conduction electron must carry with it the energy of the lattice distortion associated with the +4 valent vanadium site. As a benchmark for comparison, the entropy associated with a single electron is $k_{B} \ln (2)$, where $k_{B}$ is Boltzmann's constant, and this leads to a Seebeck coefficient of $k_{B} / q=86 \mu \mathrm{V} / \mathrm{K}$ for a Drude model electron gas. Due to lattice and defect scattering processes, electron or hole conduction within a traditional semiconductor produces Seebeck coefficients less than this magnitude. Thus, a value of $\mathrm{S} \sim-540 \mu \mathrm{V} / \mathrm{K}$ makes $\mathrm{V}_{2} \mathrm{O}_{5}$ of immediate interest for thermoelectric and thermal sensor applications.

While the Seebeck coefficient is by itself quite encouraging, $\mathrm{V}_{2} \mathrm{O}_{5}$ has relatively poor electrical conductivity because of the low mobility of its small polarons. The ZT figure for a pure film of $\mathrm{V}_{2} \mathrm{O}_{5}$ is too small to even consider for thermoelectric applications, but there are opportunities to alter the conductivity with doping. In particular, it has been found that the conductivity of $\mathrm{V}_{2} \mathrm{O}_{5}$ thin films can be increased by a factor of $\sim 10^{4}$ by sodium doping, while the degradation to the Seebeck coefficient is only a factor of $\sim 2 \cdot 5$, as shown in 


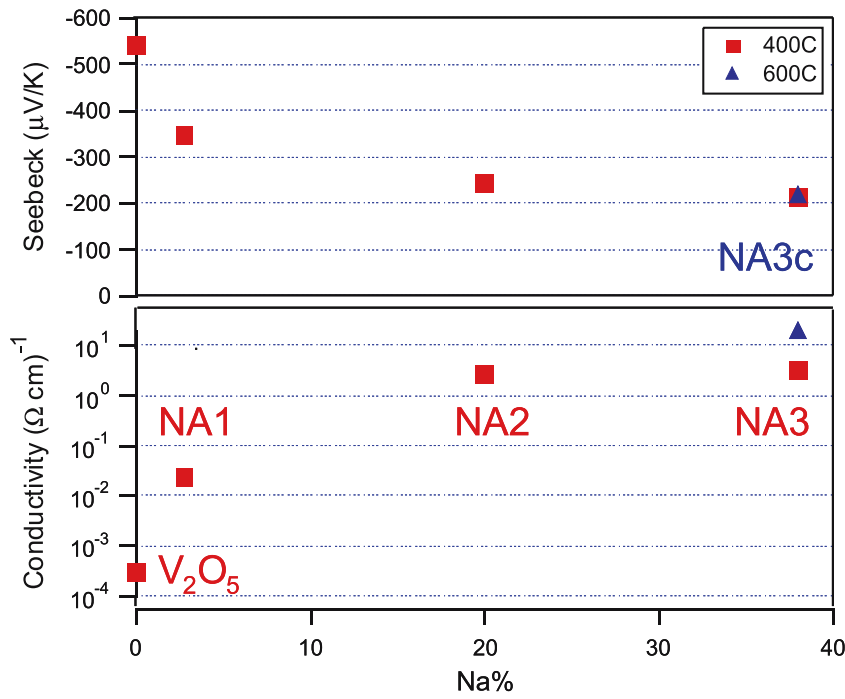

Figure 6. Seebeck coefficient and electrical conductivity of $\mathrm{Na}$ doped $\mathrm{V}_{2} \mathrm{O}_{5}$ as a function of doping level. From Iwanaga et al (2007).

figure 6 (Iwanaga et al 2007). The resulting thermopower, which is proportional to $\sigma S^{2}$, can be increased by a factor of 150 to 350 . Sodium doping increases the equilibrium density of $\mathrm{V}^{4+}$ states, and thus raises the equilibrium polaron concentration, leading to a drastically higher electrical conductivity. The degradation in the Seebeck coefficient has been found to closely follow Heike's formula, $S=\left(k_{B} / q\right) \ln \left(\mathrm{V}^{4+} / \mathrm{V}^{5+}\right)$, indicating that the transport is indeed dominated by small polaron transport (Kounavis et al 1988). This $\sim 100$-fold increase in the thermopower is encouraging, and by itself imparts these $\mathrm{V}_{2} \mathrm{O}_{5}$ thin films with the capability to be useful for thermal sensors and waste heat recovery devices. Increasing the efficiency and the ZT figure will require more research into methods to modify the thermal conductivity and increase the electrical conductivity still further.

\subsection{Potential device applications}

As discussed above, $\mathrm{V}_{2} \mathrm{O}_{5}$ thin films exhibit some remarkable physical properties that can be exploited for microelectronics and micro- to meso-scale device applications. This material becomes even more attractive when it can be fabricated by simple, inexpensive, non-toxic and scalable aqueous sol-gel techniques.

AFM nanolithography on $\mathrm{V}_{2} \mathrm{O}_{5}$ thin films has been able to produce stable and erasable colour changes along with high-contrast changes in local conductivity. The minimum size that these patterns have been written and then read out by the AFM is approximately $50 \mathrm{~nm}$. If such a $50 \mathrm{~nm} \times 50 \mathrm{~nm}$ area could be used to store one binary bit, this would lead to a data storage density of $\sim 40 \mathrm{~Gb} / \mathrm{cm}^{2}$, which is greater than what is currently possible with magnetic recording media or with sub-micron cell flash memory arrays. While this estimate obviously ignores isolation overhead, read/write architecture, and data formatting, it nevertheless indicates that there is strong potential for high density electronic read-only memory applications. Since the minimum pixel size for optical read out is approximately $2 \mu \mathrm{m}$ square, the corresponding data density for an optical RAM would be about $25 \mathrm{Mb} / \mathrm{cm}^{2}$, comparable to a conventional CD-R/W disk drive.

While the thermoelectric ZT figure-of-merit is still poor for $\mathrm{V}_{2} \mathrm{O}_{5}$ thin films, the thermopower and Seebeck coefficient are quite respectable and can be utilized for waste heat recovery 
and thermal sensors. Temperature sensors are particularly easy to implement, as a $\mathrm{V}_{2} \mathrm{O}_{5}$ thin film with ohmic contacts on each end can be directly used as a Seebeck element when it bridges across the two elements whose temperature difference is to be measured. More accurately, this type of system forms a differential thermocouple pair, with the overall temperature-tovoltage transduction factor being the difference between the Seebeck coefficient of $\mathrm{V}_{2} \mathrm{O}_{5}$ and that of the interconnect metal. But since the Seebeck coefficient of $\mathrm{V}_{2} \mathrm{O}_{5}$ is significantly larger than that of the metal interconnect, the overall transduction factor is set principally by the $\mathrm{V}_{2} \mathrm{O}_{5}$ thin film. Waste heat recovery devices are also of interest for powering small, remote instrumentation systems which must be placed near to a high temperature system. Optimization of the material and device geometry is presently on-going for this application. Also of note is that conventional thermoelectrics have Carnot efficiency limits set by the temperature of their hot side, which for most of the conventional $\mathrm{PbTe}$ or $\mathrm{Bi}_{2} \mathrm{Te}_{3}$ thermoelectric materials in use is limited to about $250^{\circ} \mathrm{C}$ before oxidation and disordering of the material causes irreversible damage. Oxide materials, such as $\mathrm{V}_{2} \mathrm{O}_{5}$, by comparison, can handle much higher hot side temperatures and operate with a higher Carnot efficiency limit. Thus, there exists considerable motivation to continue to develop the metal oxide thermoelectrics, of which $\mathrm{V}_{2} \mathrm{O}_{5}$ and its derivatives are a primary candidates.

\section{Sodium vanadium bronze, $\beta-\mathrm{Na}_{1 / 3} \mathrm{~V}_{2} \mathrm{O}_{5}$}

When sufficient sodium is introduced into the van der Waals planes of $\mathrm{V}_{2} \mathrm{O}_{5}$, it can become organized into a regular, periodic pattern to form a sodium vanadium bronze phase. The $\beta$-phase is most relevant, in which exactly half of the hexagonal interstitial sites are occupied by sodium atoms. The $\mathrm{V}_{2} \mathrm{O}_{5}$ lattice offers a number of preferred interstitial sites for intercalating alkali atoms, and among these, the most spacious are the hexagonal $E_{h}$ sites which become readily apparent when the crystal is viewed along the $c$-axis. When filled with sodium atoms such that within any plane no two adjacent interstitial sites are occupied, the hexagonal lattice of $E_{h}$ sites becomes one-third filled, leading to the structural formula $\beta-\mathrm{Na}_{1 / 3} \mathrm{~V}_{2} \mathrm{O}_{5}$ for the bronze phase.

With decreasing temperature, sodium vanadium bronze exhibits structural phase transitions at $240 \mathrm{~K}$ and $222 \mathrm{~K}$, which are associated with progressive ordering of sodium atoms into their preferred sites within the van der Waals planes, and more significantly, a MIT to a chargedordered state below $135 \mathrm{~K}$ (Obermeier et al 2002). Further, at $24 \mathrm{~K}$, it exhibits another phase transition into an antiferromagnetic state (Yamada \& Ueda 1999). Recently $\beta-\mathrm{Na}_{1 / 3} \mathrm{~V}_{2} \mathrm{O}_{5}$ has been found to exhibit a transition into a superconducting phase at $8 \mathrm{~K}$ with a pressure of $8 \mathrm{GPa}$ (Yamauchi et al 2002). At room temperature, $\beta-\mathrm{Na}_{1 / 3} \mathrm{~V}_{2} \mathrm{O}_{5}$ is a quasi-one-dimensional metal, with 100 -fold greater conductivity along the $b$-axis chains than in the $a$-directions perpendicular to them. More specifically, along the $b$-axis the conduction appears metallic, while perpendicular to the $b$-axis chains, the behaviour is semiconducting. Sodium vanadium bronze thus offers many intriguing effects for applied physics study, as well as technologically useful conduction anisotropies which might be exploited for novel interconnect and addressing schemes in nanoelectronics.

\section{Vanadium oxide nanoribbons and nanotubes}

Vanadium oxide nanotubes have been synthesized by hydrothermal methods, and these appear to be $\mathrm{V}_{2} \mathrm{O}_{5}$ with an average vanadium valence of +4.4 (Liu et al 2005). The nanotubes are only 
one of a multitude of novel structures which can be synthesized from hydrated $\mathrm{V}_{2} \mathrm{O}_{5}$ gels by hydrothermal methods. The growth of these structures can be controlled by the introduction of intercalating ions, dopants, or long-chain organic molecules, and in many cases, the $\mathrm{V}_{2} \mathrm{O}_{5}$ can be grown around the molecule, creating a ion-permeable capsule or pill which can be employed in any of a variety of biosensor applications. Other open framework $\mathrm{V}_{2} \mathrm{O}_{5}$ structures offer similar possibilities (Zavalij \& Whittingham 1999). While there have been many reports of novel $\mathrm{V}_{2} \mathrm{O}_{5}$ nanostructures, research into turning these into useful biosensors is only a beginning but offers much promise.

\section{Conclusions}

Transition metal oxides offer many unique properties for microelectronics and sensing applications, and are often too quickly disregarded in favour of silicon-based devices. Vanadium oxides in particular offer a wealth of unique properties that can be exploited for microelectronics and sensor applications, and one outstanding example of this is the use of mixed vanadium oxides in infrared imaging microbolometer arrays. This is one example of where a unique material has been able to completely revolutionize the technology, in this case the detection and imaging of mid-infrared radiation with room-temperature uncooled detector arrays. Vanadium pentoxide offers unique potential for its electrochromic, thermoelectric, and anisotropic conductivity effects. Efforts are under way at several institutions to develop these materials to the point of commercial applications. Further, the development of sodium vanadium bronzes and unique hydrothermally-synthesized $\mathrm{V}_{2} \mathrm{O}_{5}$ nanostructures may create new generations of biosensors and bioassays.

\section{References}

Adler D, Feinlaub J, Books H, Paul W 1967 Semiconductor-to-metal transitions in transition metal compounds. Phys. Rev. 155: 851-860

Balberg I, Trokman S 1975 High-contrast optical storage in $\mathrm{VO}_{2}$ films. J. Appl. Phys. 46: 2111-2119

Biermann S, Poteryaev A, Lichtenstein A I, Georges A 2005 Dynamical singlets and correlationassisted peierls transition in $\mathrm{VO}_{2}$. Phys. Rev. Lett. 94: 026404

Bullot J, Gallais O, Gauthier M, Livage J 1980 Appl. Phys. Lett. 36: 986

Cavalleri A, Dekorsy T, Chong H H W, Kieffer J C, Shoenlein R W 2004a Evidence for a structurallydriven insulator-to-metal transition in $\mathrm{VO}_{2}$ : A view from the ultrafast timescale. Phys. Rev. B, Rapid Comm. 70: 161102(R)

Cavalleri A, Chong H H W, Fourmaux S, Glover T E, Heimann P A, Kieffer J C, Mun B S, Padmore H A, Shoenlein R W 2004b Picosecond soft x-ray absorption measurement of the photoinducted insulator-to-metal transition in $\mathrm{VO}_{2}$. Phys. Rev. B69: 153106

Cavalleri A, Rini M, Chong H H W, Fourmaux S, Glover T E, Heimann P A, Kieffer J C, Shoenlein R W 2005 Band-selective measurement of electronic dynamics in $\mathrm{VO}_{2}$ using Femtosecond near edge X-ray absorption. Phys. Rev. Lett. 95: 067405

Cogan S F, Nguyen N M, Perrotti S J, Rauh R D 1989 Optical properties of electrochromic vanadium pentoxide. J. Appl. Phys. 66: 1333-1337

Eyert V, Höch K-H 1998 Electronic structure of $\mathrm{V}_{2} \mathrm{O}_{5}$ : Role of octahedral deformations. Phys. Rev. B57: 12727-12737

Haverkort M W, Hu Z, Tanaka A, Reichelt W, Streltsov S V, Korotin M A, Anisimov V I, Hsieh H H, Lin H-J, Chen C T, Khomskii D I, Tjeng L H 2005 Orbital-assisted metal-insulator transition in $\mathrm{VO}_{2}$. Phys. Rev. Lett. 95: 196404 
Hermann K, Chakrabarti A, Druzinic R, Witko M 1999 Ab-initio density functional theory studies of hydrogen absorption at the $\mathrm{V}_{2} \mathrm{O}_{5}(010)$ surface. Phys. Stat. Sol. A173: 195-208

Iwanaga S, Darling R B, Cobden D H 2005 Stable and erasable patterning of vanadium pentoxide thin films by atomic force microscope nanolithography. Appl. Phys. Lett. 86: 133113

Iwanaga S, Marciniak M, Darling R B, Ohuchi F S 2007 Thermopower and electrical conductivity of sodium-doped $\mathrm{V}_{2} \mathrm{O}_{5}$ thin films. J. Appl. Phys. 101: 123709

Jerominek H, Picard F, Vincent D 1993 Vanadium oxide films for optical switching and detection. Opt. Eng. 32: 2092-2099

Jin P, Yoshimura K, Tanemura S 1997 Dependence of microstructure and thermochromism on substrate temperature for sputter-deposited $\mathrm{VO}_{2}$ epitaxial films. J. Vac. Sci. Tech. A15: 1113-1117

Kawada I, Kimizuka N, Nakahira M 1971 Crystallographic investigations of the phase transition of $\mathrm{VO}_{2}$. J. Appl. Cryst. 4: 343-347

Kahn M S R, Khan K A, Estrada W, Granqvist C G 1991 Electrochromism and thermochromism of $\mathrm{Li}_{x} \mathrm{VO}_{2}$ thin films. J. Appl. Phys. 69: 3231-3234

Kounavis P, Vomvas A, Mytilineou E, Roilos M, Murawski L 1988 J. Phys. C21: 967

Kucharczyk D, Niklewski T 1979 Accurate X-ray determination of the lattice parameters and the thermal expansion coefficients of $\mathrm{VO}_{2}$ near the transition temperature. J. Appl. Cryst. 12: 370-373

Liu X, Täschner C, Leonhardt A, Rümmeli M H, Pichler T, Gemming T, Büchner B, Knupfer M 2005 Structural, optical, and electronic properties of vanadium oxide nanotubes. Phys. Rev. B72: 115407

Narayan J, Bhosle V M 2006 Phase transition and critical issues in structure-property correlations of vanadium oxide. J. Appl. Phys. 100: 103524

Obermeier G, Ciesla D, Klimm S, Horn S 2002 Pressure dependence of phase transitions in the quasione-dimensional metal-insulator transition system $\beta-\mathrm{Na}_{1 / 3} \mathrm{~V}_{2} \mathrm{O}_{5}$. Phys. Rev. B66: 085117

Pan M, Zhong H, Wang S-W, Li Z, Chen X, Lu W 2004 First-principle study on the chromium doping effect on the crystal structure of metallic $\mathrm{VO}_{2}$. Chem. Phys. Lett. 398: 304-307

Talledo A, Andersson A M, Granqvist C G 1991 Structure and optical absorption of $\mathrm{Li}_{y} \mathrm{~V}_{2} \mathrm{O}_{5}$ thin films. J. Appl. Phys. 69: 3261-3265

Talledo A, Granqvist C G, 1995 Electrochromic vanadium-pentoxide-based films: Structural, electrochemical, and optical properties. J. Appl. Phys. 77: 4655-4666

Wood R A, Stelzer 1992 Proc. IRIS Conf.

Wood R A, Han C J, Coole B E, Higashi R E 1992 Uncooled monolithic silicon focal plane developement Proc. IRIS Detector Speciality Group

Wood R A 2002 Semiconductors and Semimetals, 47, Ch. 3

Yamada H, Ueda Y 1999 J. Phys. Soc. Jpn. 68: 2735

Yamauchi T, Ueda Y, Môri N 2002 Pressure-induced superconductivity in $\beta-\mathrm{Na}_{0} \cdot{ }_{33} \mathrm{~V}_{2} \mathrm{O}_{5}$ beyond charge ordering. Phys. Rev. Lett. 89: 057002

Zavalij P Y, Whittingham M S 1999 Structural chemistry of vanadium oxides with open frameworks. Acta Cryst. B55: 627-633 